\title{
GLOBAL STRICHARTZ ESTIMATES FOR NONTRAPPING GEOMETRIES: ABOUT AN ARTICLE BY H. SMITH AND C. SOGGE
}

\author{
N. BURQ
}

\begin{abstract}
The purpose of this note is to present an alternative proof of a result by H. Smith and C. Sogge showing that in odd dimension of space, local (in time) Strichartz estimates and exponential decay of the local energy for solutions to wave equations imply global Strichartz estimates. Our proof allows to extend the result to the case of even dimensions of space
\end{abstract}

\section{INTRODUCTION}

Consider $\Theta \subset B(0, R) \subset \mathbb{R}^{d}$ a smooth $\left(C^{3}\right)$ obstacle, $\Omega=\Theta^{c}, g$ a smooth $\left(C^{2}\right)$ metric on $\bar{\Omega}$ equal to $g_{i, j}(x)=\delta_{i, j}$ for $|x| \geq R$, and the wave equation on $\Omega$ :

$$
\left\{\begin{array}{c}
\left(\partial_{t}^{2}-\Delta_{g}\right) u(t, x)=F(t, x) \\
\left.u\right|_{t=0}=f \in \dot{H}_{D}^{\gamma},\left.\partial_{t} u\right|_{t=0}=g \in \dot{H}_{D}^{\gamma-1} \\
\left.u\right|_{\partial \Omega}=0
\end{array}\right.
$$

where $\Delta_{g}$ is the Laplace Beltrami operator on $\Omega$, and $\dot{H}^{\gamma}, \gamma \in \mathbb{R}$ are the homogeneous Sobolev spaces associated to the square root of the Laplace operator with Dirichlet boundary conditions on $\Omega, \sqrt{-\Delta_{D}}$. In [10] H. Smith and C. Sogge show that in odd space dimensions, if the perturbation $(g, \Theta)$ is assumed to be non trapping, then local (in time) Strichartz estimates imply global (in time) Strichartz estimates for the solutions of the wave equation. The method of proof relies on the exponential decay for the local energy of solutions of the corresponding wave equation with compactly supported initial data, a localization argument on the wave cone and a result by M. Christ and A. Kiselev [5] allowing to deduce the inhomogeneous estimates from the homogeneous ones. The purpose of this note is to generalize this result to the case of even dimensions of space. In this case, the exponential decay is no more true and one has to replace it by another form of decay. In fact the decay we will use (global $L^{2}$ integrability of the local energy norm for any initial data, i.e. non necessarily compactly supported) is closely related to the

Part of this work was completed during a stay of the author at the Department of mathematics of the University of California, Berkeley, partially funded by a N.A.T.O. fellowship. I thank these two institutions for their support. 
exponential decay of the local energy used by H. Smith and C. Sogge, since the proof of our decay relies on a weaker form of the estimates used to prove the exponential decay in scattering theory.

More precisely, the result by H. Smith and C. Sogge reads as follows:

Definition $1.1(\|10\|)$. We say that $1 \leq r, s \leq 2 \leq p, q \leq+\infty$ and $\gamma$ are admissible if the following two mixed norm estimates hold

- Local Strichartz estimates. For data $f, g, F$ supported in $\{|x| \leq R\}$, and $u$ solution of (1.1), one has

$$
\begin{aligned}
\|u\|_{L_{t}^{p}\left([0,1] ; L_{x}^{q}(\Omega)\right.}+\|\left(u, \partial_{t} u \|_{L_{t}^{\infty}\left([0,1] ; \dot{H}_{D}^{\gamma} \times \dot{H}_{D}^{\gamma-1}\right.}\right. & \\
& \leq C\left(\|f\|_{\dot{H}_{D}^{\gamma}}+\|g\|_{\dot{H}_{D}^{\gamma-1}}+\|F\|_{L_{t}^{r}\left([0,1] ; L_{x}^{s}(\Omega)\right)}\right)
\end{aligned}
$$

- Global Minkovski Strichartz Estimates. For data $f, g, F$ and $u$ solution of (1.1) in the case $\Omega=\mathbb{R}^{d}$ and $g_{i, j}(x)=\delta_{i, j}$, one has

$$
\|u\|_{L_{t}^{p}\left(\mathbb{R} ; L_{x}^{q}\left(\mathbb{R}^{d}\right)\right)} \leq C\left(\|f\|_{\dot{H}_{D}^{\gamma}}+\|g\|_{\dot{H}_{D}^{\gamma-1}}+\|F\|_{L_{t}^{r}\left(\mathbb{R} ; L_{x}^{s}\left(\mathbb{R}^{d}\right)\right)}\right)
$$

In [10], H. Smith and C. Sogge show:

Theorem 1 (10, Theorem 1.1]). Assume that $d \geq 3$ is odd, $1 \leq r, s \leq 2 \leq p, q \leq+\infty$ and $\gamma$ are admissible, $p>r$ and $\gamma \leq \frac{d-1}{2}$. Then for data $f, g, F$ and $u$ solution of (1.1), one has

$$
\|u\|_{L_{t}^{p}\left(\mathbb{R} ; L_{x}^{q}(\Omega)\right.} \leq C\left(\|f\|_{\dot{H}_{D}^{\gamma}}+\|g\|_{\dot{H}_{D}^{\gamma-1}}+\|F\|_{L_{t}^{r}\left(\mathbb{R} ; L_{x}^{s}(\Omega)\right)}\right)
$$

In this article we are going to prove:

Theorem 2. Assume $d \geq 2,1 \leq r, s \leq 2 \leq p, q \leq+\infty$ and $\gamma$ are admissible, $p>2$ and $\gamma<d / 2$. Assume also if $d=2$ that $\Theta \neq \emptyset$. Then for data $f, g, F$ and $u$ solution of (1.1), then

$$
\|u\|_{L_{t}^{p}\left(\mathbb{R} ; L_{x}^{q}(\Omega)\right)} \leq C\left(\|f\|_{\dot{H}_{D}^{\gamma}}+\|g\|_{\dot{H}_{D}^{\gamma-1}}+\|F\|_{L_{t}^{r}\left(\mathbb{R} ; L_{x}^{s}(\Omega)\right)}\right)
$$

Remark 1.2. The assumption $\Theta \neq \emptyset$ is used to avoid low frequency problems. It could be replaced by a "0 is not a resonance" assumption (see section $\mathrm{Q}$ ).

As explained above, the idea in this article is to replace the exponential decay of the local energy by another (weaker) form of decay. Section 2 is hence devoted to the proof of this decay. Then in section 3 we prove Theorem 2 .

Remark 1.3. At the final stage of this work, I have learned that the results of this article have been obtained independantly (by a slightly different method) by Jason Metcalfe [9] 


\section{Global $L^{2}$-Integrability of the LOCAL ENERGY}

We begin first by recalling the definition of the homogeneous Sobolev spaces (in the boundary case) $\dot{H}_{D}^{s}$. Remark that we shall restrict ourselves to the case $s<d / 2$, hence the multiplication by a smooth function $\xi \in C_{0}^{\infty}\left(\mathbb{R}^{d}\right)$ is continuous from $\dot{H}^{s}\left(\mathbb{R}^{d}\right)$ to $H^{s}\left(\mathbb{R}^{d}\right)$ and the two norms are equivalent on functions with fixed compact support. Take $R$ large enough so that $\Theta \subset B(0, R)$ and $g_{i, j}(x)=\delta_{i, j}$ for $|x| \geq R$. Fix $\beta \in C_{0}^{\infty}\left(\mathbb{R}^{d}\right)$ equal to 1 on $\{|x| \leq R\}$, and define for $s \geq 0 \dot{H}_{D}^{s}$ to be the set of functions $f \in H_{\text {loc }}^{s}(\Omega)$ such that

$$
\|f\|_{\dot{H}_{D}^{s}}^{2}=\|\beta f\|_{H^{s}(\Omega)}^{2}+\|(1-\beta) f\|_{\dot{H}^{s}\left(\mathbb{R}^{d}\right)}^{2}
$$

is bounded and which satisfy the following compatibility conditions:

$$
\left.\Delta_{g}^{j} f\right|_{\partial \Omega}=0, \forall j ; s-2 j>\frac{1}{2} .
$$

For $s<0$, define $\dot{H}_{D}^{s}=\left(\dot{H}_{D}^{-s}\right)^{\prime}$ Remark that on $\dot{H}_{D}^{s}$, the norms $\left\|\left(-\Delta_{g}\right)^{s / 2} f\right\|_{L^{2}}$ and $\|f\|_{\dot{H}_{D}^{s}}$ are equivalent (it is clear for $s$ an even integer and follows in general by interpolation and duality). Denote by $\dot{\mathcal{H}}^{s}=\dot{H}_{D}^{s} \times \dot{H}_{D}^{s-1}$ and $\mathcal{H}^{s}=H_{D}^{s} \times H_{D}^{s-1}$.

In this part we are going to prove:

Theorem 3. Consider $\chi \in C_{0}^{\infty}\left(\mathbb{R}^{d}\right)$. Assume that $d \geq 2$ and if $d=2$ that $\Theta \neq \emptyset$. Then For data $f, g, F$ such that $F$ is supported in $B_{x}(0, R) \cap \Omega$ and $u$ solution of (1.1), one has

$$
\left\|\left(\chi u, \chi \partial_{t} u\right)\right\|_{L_{t}^{2}\left(\mathbb{R} ; \dot{H}_{D}^{\gamma} \times \dot{H}_{D}^{\gamma-1}\right)} \leq C\left(\|f\|_{\dot{H}_{D}^{\gamma}}+\|g\|_{\dot{H}_{D}^{\gamma-1}}+\|F\|_{L_{t}^{2}\left(\mathbb{R} ; \dot{H}_{D}^{\gamma-1}\right)}\right)
$$

Remark 2.1. Remark that in the result above only $F$ (and neither $f$ nor $g$ ) is assumed to be compactly supported.

Remark 2.2. As will appear below, the proof of this result is closely related to the proof of the exponential decay of the local energy (for compactly supported data). In fact we will use some estimates on the resolvent weaker than the estimates required to prove this exponential decay. The result above can be transposed to the framework of Schrödinger equations. It gives then a (global in time) "smoothing effect" 4 .

Remark 2.3. For $d=2, \gamma \leq 1 / 2$ and $g_{i, j}(x)=\delta_{i, j}$, for any $u$ solution of (1.1) with $F=0$, we have (see [10, Lemma 2.2])

$$
\left\|\left(\chi u, \chi \partial_{t} u\right)\right\|_{L_{t}^{2}\left(\mathbb{R} ; \dot{H}_{D}^{\gamma} \times \dot{H}_{D}^{\gamma-1}\right)} \leq C\left(\|f\|_{\dot{H}_{D}^{\gamma}}+\|g\|_{\dot{H}_{D}^{\gamma-1}}\right)
$$

To prove Theorem 3, we first show that by a $T T^{*}$ argument it is enough to prove a non homogeneous estimate. Indeed denote by $A=i\left(\begin{array}{cc}0 & - \text { Id } \\ -\Delta & 0\end{array}\right)$ the self adjoint operator 
on $\mathcal{H}=H_{0}^{1}(\Omega) \times L^{2}(\Omega)$ with domain $\mathcal{D}=\left\{(u, v) \in \mathcal{H} \cap H^{2}(\Omega) \times H_{0}^{1}(\Omega)\right\}$. The solution of (1.1) with $F=0$ is given by

$$
\left(\begin{array}{c}
u \\
\partial_{t} u
\end{array}\right)=e^{i t A}\left(\begin{array}{c}
f \\
g
\end{array}\right)
$$

Remark that the operator $e^{i t A}$ is a group of isometries on $\dot{\mathcal{H}}_{D}^{s}$ (equipped with the norm $\left.\left\|A^{s} f\right\|_{L^{2}}\right)$.

Denote by $T=\chi e^{i t A}$. The continuity of $T$ from $\dot{\mathcal{H}}^{s}$ to $L^{2}\left(\mathbb{R}_{t} ; \mathcal{H}^{s}\right)$ is equivalent to the continuity of $T^{*}$ from $L^{2}\left(\mathbb{R}_{t} ; \mathcal{H}^{s}\right)$ to $\dot{\mathcal{H}}^{s}$. But

$$
T^{*} G=\int_{s \in \mathbb{R}} e^{-i s A} \chi G(s, \cdot) d s
$$

and the continuity of $T^{*}$ is in turn equivalent to the continuity of $T T^{*}$ from $L^{2}\left(\mathbb{R}_{t} ; \dot{\mathcal{H}}^{s}\right)$ to $L^{2}\left(\mathbb{R}_{t} ; \mathcal{H}^{s}\right)$. Finally

$$
T T^{*} G(t, \cdot)=\int_{s \in \mathbb{R}} \chi e^{i(t-s) A} \chi G(s, \cdot) d s=\int_{s<t}+\int_{t<s}
$$

And it sufficies to prove the continuity of any of the two operators above, say for example the continuity of $\chi T T_{1}^{*} \chi$ with

$$
T T_{1}^{*} G(t, \cdot)=\int_{s<t} e^{i(t-s) A} G(s, \cdot) d s
$$

Remark that this latter result implies in turn the nonhomogeneous part of Theorem 3 . Suppose that $G$ is supported in $\{s>0\}$ (which by density and translation invariance is possible). Then $U=T T_{1}^{*} G$ satisfies the equation

$$
\left(i \partial_{t}+A\right) U=\chi G(t, \cdot),\left.U\right|_{t<0}=0
$$

The Fourier transforms with respect to $t$ of $U$ and $G$ are holomorphic in the half plane $\operatorname{Im} z<0$ (due to the support property) and satisfy there

$$
(A-z) \widehat{U}=\chi \widehat{G}
$$

Taking $z=x-i \varepsilon$ and letting $\varepsilon>0$ tend to 0 , Theorem 3 is a consequence of the fact that the Fourier transform is an isometry on $L^{2}\left(\mathbb{R}_{t} ; \mathcal{H}\right)$ if $\mathcal{H}$ is a Hilbert space and of

Proposition 2.4. Under the assumptions of Theorem \&, the resolvent $\chi(A-(x-i \varepsilon))^{-1} \chi$ is for $x \in \mathbb{R}$ and $0<|\varepsilon|<1$, uniformly bounded on $\mathcal{H}_{D}^{s}$.

We are going to deduce Proposition 2.4 from the classical result: 
Proposition 2.5. Suppose that the obstacle $\Theta$ is non trapping. Then the resolvent of the operator $\Delta_{D},\left(-\Delta_{D}-\lambda\right)^{-1}$ (which is analytic in $\mathbb{C} \backslash \mathbb{R}^{+}$) satisfies:

$$
\begin{aligned}
& \forall \chi \in C_{0}^{\infty}\left(\mathbb{R}^{2}\right), \exists C>0 ; \forall \lambda \in \mathbb{R}, 0<\varepsilon<<1, \\
& \left\|\chi\left(-\Delta_{D}-(\lambda \pm i \varepsilon)\right)^{-1} \chi\right\|_{L^{2} \rightarrow L^{2}} \leq \frac{C}{1+\sqrt{|\lambda|}}
\end{aligned}
$$

Remark 2.6. Proposition 2.5 was proven for $|\lambda|>>1$ in greater generalities by Lax and Phillips [6], Melrose and Sjöstrand [7, 8]), Vainberg[13], Tang Zworski [11] (see also [3] for a self contained proof which joined with the results in 1] would relax the smoothness assumption required in these papers to a $C^{2}$ assumption). The proof for $|\lambda|<<1$ can be found in [2, Annexe B.2] (see also [12, 13]). Remark that in [2] the Poincaré inequality is used to control the local $L^{2}$-norm by the local $L^{2}$-norm of the gradient of a function (wich is why $\Theta \neq \emptyset$ is required). However, the following inequality

$$
\forall \beta<\frac{d}{2}-1, \forall u \in C_{0}^{\infty}\left(\mathbb{R}^{d}\right),\left\|\frac{u}{r\left(1+r^{2}\right)^{\beta / 2}}\right\|_{L^{2}} \leq C\left\|\frac{\nabla u}{\left(1+r^{2}\right)^{\beta / 2}}\right\|_{L^{2}}
$$

allows to handle the argument for $d \geq 4$ (and the result is standard in odd dimensions).

Since

$$
(A-z)^{-1}=\left(\begin{array}{cc}
-z\left(\Delta_{g}+z^{2}\right)^{-1} & i\left(\Delta_{g}+z^{2}\right)^{-1} \\
i \Delta_{g}\left(\Delta_{g}+z^{2}\right)^{-1} & -z\left(\Delta_{g}+z^{2}\right)^{-1}
\end{array}\right)
$$

to prove Proposition 2.4 we have to estimate uniformly for any $s \in \mathbb{R}$

$$
\begin{aligned}
& \left\|\chi(1+|z|)\left(\Delta_{g}+z^{2}\right)^{-1} \chi\right\|_{H_{D}^{s} \rightarrow H_{D}^{s}} \\
& \left\|\chi\left(\Delta_{g}+z^{2}\right)^{-1} \chi\right\|_{H_{D}^{s} \rightarrow H_{D}^{s+1}} \\
& \left\|\chi \Delta_{g}\left(\Delta_{g}+z^{2}\right)^{-1} \chi\right\|_{H_{D}^{s} \rightarrow H_{D}^{s-1}}
\end{aligned}
$$

Remark that using duality and interpolation it is enough to do this for $s \in \mathbb{N}$.

For $s=0$, (2.14) is Proposition 2.5 and if $u=\left(\Delta_{g}+z^{2}\right)^{-1} \chi f$, we have

$$
\begin{aligned}
\int_{\Omega} \chi f \chi \bar{u} & =\int_{\Omega}\left(\Delta+z^{2}\right) u \chi \bar{u} \\
& =\int_{\Omega}-\chi|\nabla u|^{2}-\nabla \chi \cdot \nabla u \bar{u}+z^{2} \chi|u|^{2}
\end{aligned}
$$

which by Cauchy Schwartz implies (using (2.14) for $s=0$ and a different $\chi$ ) easily (2.15) for $s=0$. By duality we get also (2.15) for $s=-1$. Applying $\Delta_{g}$ to $\chi\left(\Delta_{g}+z^{2}\right)^{-1} \chi$ and commuting with $\chi$ we obtain by induction (2.15) for $s \in \mathbb{N}$ (hence by duality and 
interpolation for any $s$ ). Applying again $\Delta_{g}$ we deduce (2.16) for any $s$. Finally, remark that

$$
\chi \Delta_{g}\left(\Delta_{g}+z^{2}\right)^{-1} \chi=\chi \operatorname{Id} \chi+\chi z^{2}\left(\Delta_{g}+z^{2}\right)^{-1} \chi
$$

from which we estimate uniformly

$$
\left\|\chi z^{2}\left(\Delta_{g}+z^{2}\right)^{-1} \chi\right\|_{H_{D}^{s} \rightarrow H_{D}^{s-1}}
$$

Interpolating this latter inequality with $(2.15)_{s}$ we get $(2.14)_{s}$.

\section{From Energy decay to global Strichartz estimates}

In this section we are going to show how the local (in time) Strichartz estimates and the global (in time) $L^{2}$ - energy decay imply global (in time) Strichartz estimates. We consider firstly the homogeneous case $(F=0)$ and we also assume that $\gamma \leq 1 / 2$ if $d=2$.

Consider $\chi \in C_{0}^{\infty}\left(\mathbb{R}^{d}\right)$ equal to 1 on $B(0, R), u$ solution of (1.1) with $F=0$ and $v=\chi(x) u, w=(1-\chi)(x) u$ solutions of

$$
\begin{gathered}
\left\{\begin{array}{c}
\left(\partial_{t}^{2}-\Delta_{g}\right) v(t, x)=-\left[\Delta_{g}, \chi\right] u \\
\left.v\right|_{t=0}=\chi f \in \dot{H}^{\gamma} \\
\left.\partial_{t} v\right|_{t=0}=\chi g \in \dot{H}_{D}^{\gamma-1} \\
\left.v\right|_{\partial \Omega}=0
\end{array}\right. \\
\left\{\begin{array}{c}
\left(\partial_{t}^{2}-\Delta_{g}\right) w(t, x)=\left(\partial_{t}^{2}-\Delta_{0}\right) w(t, x)=-\left[\Delta_{0}, \chi\right] u \\
\left.w\right|_{t=0}=(1-\chi) f \in \dot{H}^{\gamma}, \\
\left.\partial_{t} w\right|_{t=0}=(1-\chi) g \in \dot{H}^{\gamma-1}
\end{array}\right.
\end{gathered}
$$

First we show that $w$ satisfies global Strichartz stimates. Since $w$ is a solution of the free wave equation (because it is supported in the set where $g_{i, j}(x)=\delta_{i, i}$ ), the contributions of $(1-\chi) f$ and $(1-\chi) g$ are handled using (1.3). Consequently it sufficies to study $\widetilde{w}$ the solution of

$$
\left\{\begin{aligned}
\left(\partial_{t}^{2}-\Delta_{0}\right) \widetilde{w}(t, x) & =-\left[\Delta_{0}, \chi\right] u \\
\left.\widetilde{w}\right|_{t=0} & =0 \\
\left.\partial_{t} \widetilde{w}\right|_{t=0} & =0
\end{aligned}\right.
$$

For this we use the following result by M. Christ and A. Kiselev [5]:

Theorem 4 (M. Christ and A. Kiselev). Consider a bounded operator $T: L^{p}\left(\mathbb{R} ; B_{1}\right) \rightarrow$ $L^{q}\left(\mathbb{R} ; B_{2}\right)$ given by a locally integrable kernel $K(t, s)$ with value operators from $B_{1}$ to $B_{2}$ 
where $B_{1 ; 2}$ are Banach spaces. Suppose that $p<q$. Then the operator

$$
\widetilde{T} f(t)=\int_{s<t} K(t, s) f(s) d s
$$

is bounded from $L^{p}\left(\mathbb{R} ; B_{1}\right)$ to $L^{q}\left(\mathbb{R} ; B_{2}\right)$ by

$$
\|\widetilde{T}\|_{L^{p}\left(\mathbb{R} ; B_{1}\right) \rightarrow L^{q}\left(\mathbb{R} ; B_{2}\right)} \leq\left(1-2^{\left.-p^{-1}-q^{-1}\right)^{-1}\|T\|_{L^{p}\left(\mathbb{R} ; B_{1}\right) \rightarrow L^{q}\left(\mathbb{R} ; B_{2}\right)}}\right.
$$

According to (3.3),

$$
\left(\widetilde{w}, \partial_{t} \widetilde{w}\right)(t, x)=\int_{0}^{t} e^{i(t-s) A_{0}}-\left[\Delta_{g}, \chi\right] u(s, \cdot) d s
$$

According to Theorem 3 (and Remark 2.3 since $\gamma \leq 1 / 2$ if $d=2$ ) applied to the free wave operator, for any $\chi \in C_{0}^{\infty}\left(\mathbb{R}^{d}\right)$ the operator $T_{0}=\chi e^{i t A_{0}} \operatorname{maps} \dot{H}^{\gamma} \times \dot{H}^{\gamma-1}$ into $L^{2}\left(\mathbb{R}_{t}^{+} ; \dot{H}^{\gamma} \times \dot{H}^{\gamma-1}\right)$ and consequently its adjoint

$$
T_{0}^{*} G=\int_{0}^{\infty} e^{-i s A_{0}} \chi G d s
$$

maps $L^{2}\left(\mathbb{R}_{t}^{+} ; \dot{H}^{\gamma} \times \dot{H}^{\gamma-1}\right)$ into $\dot{H}^{\gamma} \times \dot{H}^{\gamma-1}$ and since

$$
\left(\widetilde{w}, \partial_{t} \widetilde{w}\right)=-\int_{0}^{t} e^{i(t-s) A_{0}}\left(0,\left[\Delta_{g}, \chi\right] u\right) d s,
$$

the global Strichartz estimate follows from Theorem 4 (here we are speaking about adjoints relative to the $L^{2}$ in time and $\dot{H}^{\gamma} \times \dot{H}^{\gamma-1}$ in space norms).

We come back to the analysis of $v=\chi u$. Consider $\varphi \in C_{0}^{\infty}(] 0,1[)$ equal to 1 on $[1 / 4,3 / 4]$. Denote by $v_{n}=\varphi(t-n / 2) v$ solution of

$$
\left\{\begin{aligned}
\left(\partial_{t}^{2}-\Delta_{g}\right) v_{n}(t, x) & =-\varphi(t-n / 2)\left[\Delta_{g}, \chi\right] u+\left[\partial_{t}^{2}, \varphi(t-n / 2)\right] \chi u \\
& =u_{n}(t, x) \\
\left.v_{n}\right|_{t<n / 2} & =0 \\
\left.\partial_{t} v_{n}\right|_{t<n / 2} & =0 \\
v_{\left.n\right|_{\partial \Omega}} & =0
\end{aligned}\right.
$$

With, according to Theorem 3 ,

$$
\sum_{n \in \mathbb{Z}}\left\|u_{n}\right\|_{L^{2}\left(\mathbb{R}_{t} ; \dot{H}_{D}^{\gamma-1}\right)}^{2} \leq C\left(\|f\|_{\dot{H}_{D}^{\gamma}}^{2}+\|g\|_{\dot{H}_{D}^{\gamma-1}}^{2}\right)
$$

According to the (local in time) Strichartz inequality (1.2) (and Minkovski inequality), we obtain

$$
\left\|v_{n}\right\|_{L_{t}^{p}\left(\mathbb{R}_{t} ; L_{x}^{q}(\Omega)\right)}+\|\left(v_{n}, \partial_{t} v_{n} \|_{L_{t}^{\infty}\left([0,1] ; \dot{H}_{D}^{\gamma} \times \dot{H}_{D}^{\gamma-1}\right)} \leq C\left(\left\|u_{n}\right\|_{L_{t}^{1}\left([-1+n / 2,2+n / 2] ; H_{x}^{\gamma-1}(\Omega)\right)}\right)\right.
$$


Since

$$
\|v\|_{L_{t}^{p}\left(\mathbb{R} ; L_{x}^{q}(\Omega)\right)}^{p} \sim \sum_{n \in \mathbb{Z}}\left\|v_{n}\right\|_{L_{t}^{p}\left(\mathbb{R}_{t} ; L_{x}^{q}(\Omega)\right.}^{p}
$$

and since $p \geq 2$, we obtain, using (3.10) and (3.11)

$$
\begin{aligned}
\|v\|_{L_{t}^{p}\left(\mathbb{R} ; L_{x}^{q}(\Omega)\right)}^{p} & \leq \sum_{n \in \mathbb{Z}}\left\|v_{n}\right\|_{L_{t}^{p}\left(\mathbb{R}_{t} ; L_{x}^{q}(\Omega)\right)}^{p} \leq C \sum_{n \in \mathbb{Z}}\left\|u_{n}\right\|_{L_{t}^{1} ; H_{x}^{\gamma-1}(\Omega)}^{p} \\
& \leq C\left(\sum_{n \in \mathbb{Z}}\left\|u_{n}\right\|_{L_{t}^{2} ; H_{x}^{\gamma-1}(\Omega)}^{2}\right)^{p / 2} \leq C\left(\|f\|_{\dot{H}_{D}^{\gamma}}^{2}+\|g\|_{\dot{H}_{D}^{\gamma-1}}^{2}\right)^{p / 2}
\end{aligned}
$$

which proves Theorem 2 for $F=0$. As recognized by H. Smith and C. Sogge [10], another application of Theorem 1 gives the general case.

Finally, if $d=2$ we have to get rid of the assumption $\gamma \leq 1 / 2$. Interpolating between (2.4) and the energy estimate (for any $\gamma<1$ )

$$
\left\|\left(\chi u, \chi \partial_{u}\right)\right\|_{L^{\infty}\left(\mathbb{R}_{t} ; \dot{H}_{D}^{\gamma} \times \dot{H}^{\gamma-1}\right)} \leq\|(f, g)\|_{L^{\infty}\left(\mathbb{R}_{t} ; \dot{H}_{D}^{\gamma} \times \dot{H}^{\gamma-1}\right)}
$$

we obtain for any $q>1 /(1-\gamma)$

$$
\left\|\left(\chi u, \chi \partial_{t} u\right)\right\|_{L^{q}\left(\mathbb{R}_{t} ; \dot{H}_{D}^{\gamma} \times \dot{H}^{\gamma-1}\right)} \leq\|(f, g)\|_{L^{\infty}\left(\mathbb{R}_{t} ; \dot{H}_{D}^{\gamma} \times \dot{H}^{\gamma-1}\right)}
$$

using this to estimate $\widetilde{w}$ and Christ-Kiselev Theorem we can conclude as above provided $p>1 /(1-\gamma)$. Finally we remark that this latter condition is automatically fulfilled due to the scaling condition $\frac{1}{p}+\frac{d}{q}=\frac{d}{2}-\gamma$ which is necessary for (1.2) to hold.

\section{REFERENCES}

[1] N. Burq. Contrôle de l'équation des ondes dans des ouverts peu réguliers. Asymptotic Analysis, 14:157191, 1997.

[2] N. Burq. Décroissance de l'énergie locale de l'équation des ondes pour le problème extérieur et absence de résonance au voisinage du réel. Acta Mathematica, 180:1-29, 1998.

[3] N. Burq. Semi-classical estimates for the resolvent in non trapping geometries. Int. Math. Res. Notices, 5:221-241, 2002.

[4] N. Burq, P. Gerard, and N. Tzvetkov. Large data global well-posedness for non-linear schrödinger equations in exterior domains. in preparation, 2002.

[5] Michael Christ and Alexander Kiselev. Maximal functions associated to filtrations. J. Funct. Anal., 179(2):409-425, 2001.

[6] P. D. Lax and R. S. Phillips. Scattering theory. Number 26 in Pure and Applied Mathematics. Academic Press, 2 edition, 1989.

[7] R.B. Melrose and J. Sjöstrand. Singularities of boundary value problems I. Communications in Pure Applied Mathematics, 31:593-617, 1978.

[8] R.B. Melrose and J. Sjöstrand. Singularities of boundary value problems II. Communications in Pure Applied Mathematics, 35:129-168, 1982.

[9] J. Metcalfe. Global strichartz estimates for solutions to the wave equation exterior to a convex obstacle. preprint, 2002. 
[10] Hart F. Smith and Christopher D. Sogge. Global Strichartz estimates for nontrapping perturbations of the Laplacian. Comm. Partial Differential Equations, 25(11-12):2171-2183, 2000.

[11] Siu-Hung Tang and Maciej Zworski. Resonance expansions of scattered waves. Comm. Pure Appl. Math., 53(10):1305-1334, 2000.

[12] B. R. Vainberg. On the analytical properties of the resolvent for a certain class of operator pencils. Math. USSR Sbornik, 6(2):241-273, 1968.

[13] B. R. Vainberg. Asymptotic methods in equations of matheatical physics. Gordon and Breach, New York, 1988.

Mathématiques, BÂt. 425, Université Paris Sud Orsay, 91405 Orsay Cedex, France

E-mail address: nicolas.burq@math.u-psud.fr

$U R L$ : www.math.u-psud.fr/ burq 